\title{
On the integer sets with the same representation functions
}

\author{
Kai-Jie Jiao, ${ }^{1}$ Csaba Sándor, ${ }^{2 *}$ Quan-Hui Yang ${ }^{1 \dagger}$ and Jun-Yu Zhou ${ }^{1}$ \\ 1. School of Mathematics and Statistics, Nanjing University of Information \\ Science and Technology, Nanjing 210044, China
}

2. Institute of Mathematics, Budapest University of Technology and Economics, and MTA-BME Lendület Arithmetic Combinatorics Research Group, ELKH, H-1529 B.O. Box, Hungary

\begin{abstract}
Let $\mathbb{N}$ be the set of all nonnegative integers. For $S \subseteq \mathbb{N}$ and $n \in \mathbb{N}$, let $R_{S}(n)$ denote the number of solutions of the equation $n=s_{1}+s_{2}, s_{1}, s_{2} \in S$ and $s_{1}<s_{2}$. Let $A$ be the set of all nonnegative integers which contain an even number of digits 1 in their binary representations and $B=\mathbb{N} \backslash A$. Put $A_{l}=A \cap\left[0,2^{l}-1\right]$ and $B_{l}=B \cap\left[0,2^{l}-1\right]$. In 2017, Kiss and Sándor proved that, if $C \cup D=[0, m]$, $0 \in C$ and $C \cap D=\{r\}$, then $R_{C}(n)=R_{D}(n)$ for every positive integer $n$ if and only if there exists an integer $l \geq 1$ such that $r=2^{2 l}-1, m=2^{2 l+1}-2, C=A_{2 l} \cup\left(2^{2 l}-1+B_{2 l}\right)$ and $D=B_{2 l} \cup\left(2^{2 l}-1+A_{2 l}\right)$. This solved a problem of Chen and Lev. In this paper, we prove that, if $C \cup D=[0, m] \backslash\{r\}$ with $0<r<m, C \cap D=\emptyset$ and $0 \in C$, then $R_{C}(n)=R_{D}(n)$ for any nonnegative integer $n$ if and only if there exists an integer $l \geq 2$ such that $m=2^{l}, r=2^{l-1}, C=A_{l-1} \cup\left(2^{l-1}+1+B_{l-1}\right)$ and $D=B_{l-1} \cup\left(2^{l-1}+1+A_{l-1}\right)$.
\end{abstract}

Keywords: Sárkozy's problem, Thue-Morse sequence, representation function.

\section{Introduction}

Let $\mathbb{N}$ be the set of all nonnegative integers. For $S \subseteq \mathbb{N}$ and $n \in \mathbb{N}$, let $R_{S}(n)$ denote the number of solutions of the equation $n=s_{1}+s_{2}, s_{1}, s_{2} \in S$ and $s_{1}<s_{2}$. Sárközy asked whether there exist two sets $U$ and $V$ of nonnegative integers with infinite symmetric difference and $R_{U}(n)=R_{V}(n)$ for all large enough integers $n$. Dombi [5] answered this problem affirmatively by using the Thue-Morse sequence. Later Lev [7], Sándor [9], and Tang [10] gave a simple proof of this result respectively. For related results, one can refer to [4], [12]-[14].

Let $A$ be the set of all nonnegative integers which contain an even number of digits 1 in their binary representations and $B=\mathbb{N} \backslash A$. For any positive integer $l$, let $A_{l}=A \cap\left[0,2^{l}-1\right]$ and $B_{l}=B \cap\left[0,2^{l}-1\right]$. In 2017, Kiss and Sándor [6] proved that if $C$ and $D$ are two sets of nonnegative integers such that $C \cup D=[0, m]$, $C \cap D=\emptyset$ and $0 \in C$, then $R_{C}(n)=R_{D}(n)$ for every positive integer $n$ if and only if there exists a positive integer $l$ such that $C=A_{l}$ and $D=B_{l}$. In 2016, Tang [11] proved that for any given integer $m$, there do not exist subsets $C, D \subseteq \mathbb{N}$ with $\mathbb{N}=C \cup D$ and $C \cap D=\{k m: k \in \mathbb{N}\}$ such that $R_{C}(n)=R_{D}(n)$ for all sufficiently large integers $n$.

For any integer $r$ and $m$, we define $r+m \mathbb{N}=\{r+m a: a \in \mathbb{N}\}$. Chen and Lev [1] proved that, for any given positive integer $l$, there exist two subsets $C, D \subseteq \mathbb{N}$ with $C \cup D=\mathbb{N}$ and $C \cap D=2^{2 l}-1+\left(2^{2 l+1}-1\right) \mathbb{N}$ such that $R_{C}(n)=R_{D}(n)$ for every positive integer $n$. They also posed the following two problems.

Problem 1. Given that $R_{C}(n)=R_{D}(n)$ for every positive integer $n, C \cup D=\mathbb{N}$, and $C \cap D=r+m \mathbb{N}$ with integers $r \geq 0$ and $m \geq 2$, must there exist an integer $l \geq 1$ such that $r=2^{2 l}-1, m=2^{2 l+1}-1$ ?

Problem 2. Given that $R_{C}(n)=R_{D}(n)$ for every positive integer $n, C \cup D=[0, m]$, and $C \cap D=\{r\}$ with integers $r \geq 0$ and $m \geq 2$, must there exist an integer $l \geq 1$ such that $r=2^{2 l}-1, m=2^{2 l+1}-2$, $C=A_{2 l} \cup\left(2^{2 l}-1+B_{2 l}\right)$ and $D=B_{2 l} \cup\left(2^{2 l}-1+A_{2 l}\right)$ ?

*Email: csandor@math.bme.hu. This author was supported by the OTKA Grant No. K129335.

${ }^{\dagger}$ Email: yangquanhui01@163.com. 
In 2017, Kiss and Sándor solved Problem 2 affirmatively. Later, Li and Tang [8], Chen, Tang and Yang [3] solved Problem 1 under the assumption $0 \leq r<m$. Recently, Chen and Chen [2] solved Problem 1 affirmatively.

In this paper, we consider two sets $C$ and $D$ satisfying $C \cup D=[0, m] \backslash\{r\}$ and $C \cap D=\emptyset$. We prove the following result.

Theorem 1.1. Let $C$ and $D$ be two sets of nonnegative integers such that $C \cup D=[0, m] \backslash\{r\}$ with $0<r<m$, $C \cap D=\emptyset$ and $0 \in C$. Then for any nonnegative integer $n, R_{C}(n)=R_{D}(n)$ if and only if there exists an integer $l \geq 2$ such that $m=2^{l}, r=2^{l-1}, C=A_{l-1} \cup\left(2^{l-1}+1+B_{l-1}\right)$ and $D=B_{l-1} \cup\left(2^{l-1}+1+A_{l-1}\right)$.

\section{Preliminary Lemmas}

Lemma 2.1. For positive integers $0<r_{1}<r_{2}<\cdots<r_{s} \leq m$, there exist at most a pair of sets $(C, D)$ such that $C \cup D=[0, m] \backslash\left\{r_{1}, r_{2}, \ldots, r_{s}\right\}, 0 \in C, C \cap D=\emptyset$ and $R_{C}(k)=R_{D}(k)$ for all $k \leq m$.

Proof. Suppose that there exist two pair of sets $\left(C_{1}, D_{1}\right),\left(C_{2}, D_{2}\right)$ satisfying the condition of Lemma 2.1. Let $v$ be the minimal nonnegative integer such that $\chi_{C_{1}}(v) \neq \chi_{C_{2}}(v)$. Then $C_{1} \cap[0, v-1]=C_{2} \cap[0, v-1]$ and $R_{C_{1}}(v) \neq R_{C_{2}}(v)$ since $0 \in C_{1}, C_{2}$. By $R_{C_{1}}(v)=R_{D_{1}}(v)$ and $R_{C_{2}}(v)=R_{D_{2}}(v)$, it suffices to prove that $R_{D_{1}}(v)=R_{D_{2}}(v)$ for a contradiction.

Since $0 \in C$ and $C \cap D=\emptyset$, it follows that $0 \notin D$, and so

$$
R_{D_{1}}(v)=\left|\left\{\left(d, d^{\prime}\right): d<d^{\prime}<v, d, d^{\prime} \in D_{1}, d+d^{\prime}=v\right\}\right| .
$$

Let $t$ be an integer with $0 \leq t \leq s$ such that $r_{t}<v<r_{t+1}$. Here we define $r_{0}=0, r_{s+1}=+\infty$.

Since

$$
[0, v-1] \backslash\left\{r_{1}, r_{2}, \ldots, r_{t}\right\}=\left(C_{1} \cap[0, v-1]\right) \cup\left(D_{1} \cap[0, v-1]\right),
$$

it follows that

$D_{1} \cap[0, v-1]=\left([0, v-1] \backslash\left\{r_{1}, r_{2}, \ldots, r_{t}\right\}\right) \backslash\left(C_{1} \cap[0, v-1]\right)=[0, v-1] \backslash\left(\left(C_{1} \cap[0, v-1]\right) \cup\left\{r_{1}, r_{2}, \ldots, r_{s}\right\}\right)$.

Similarly,

$$
D_{2} \cap[0, v-1]=[0, v-1] \backslash\left(\left(C_{2} \cap[0, v-1]\right) \cup\left\{r_{1}, r_{2}, \ldots, r_{t}\right\}\right) .
$$

Hence $D_{1} \cap[0, v-1]=D_{2} \cap[0, v-1]$, and so $R_{D_{1}}(v)=R_{D_{2}}(v)$.

Remark 2.1. If $\left\{r_{1}, r_{2}, \ldots, r_{s}\right\}=\emptyset$, then Lemma 2.1 also holds.

Lemma 2.2. Suppose that $C, D$ are two distinct sets satisfying $C \cup D=[0, m] \backslash\left\{r_{1}, \ldots, r_{s}\right\}, C \cap D=\emptyset$ and $R_{C}(n)=R_{D}(n)$ for any positive integer $n$. If $C^{\prime}=m-C, D^{\prime}=m-D$, then $C^{\prime} \cup D^{\prime}=[0, m] \backslash$ $\left\{m-r_{1}, \ldots, m-r_{s}\right\}, C^{\prime} \cap D^{\prime}=\emptyset$, and $R_{C^{\prime}}(n)=R_{D^{\prime}}(n)$ for all nonnegative integers $n$.

Proof. For $k=0,1, \ldots, 2 m$,

$$
\begin{aligned}
R_{C^{\prime}}(k) & =\left|\left\{\left(c, c^{\prime}\right): c<c^{\prime}, c, c^{\prime} \in C^{\prime}, c+c^{\prime}=k\right\}\right| \\
& =\left|\left\{\left(c, c^{\prime}\right): c<c^{\prime}, m-c, m-c^{\prime} \in C, c+c^{\prime}=k\right\}\right| \\
& =\left|\left\{\left(m-c, m-c^{\prime}\right): c<c^{\prime}, m-c, m-c^{\prime} \in C, 2 m-\left(c+c^{\prime}\right)=2 m-k\right\}\right| \\
& =R_{C}(2 m-k) .
\end{aligned}
$$

Similarly, $R_{D^{\prime}}(k)=R_{D}(2 m-k)$. Since $R_{C}(2 m-k)=R_{D}(2 m-k)$, it follows that $R_{C^{\prime}}(k)=R_{D^{\prime}}(k)$.

Lemma 2.3. (See [6, Claim 3]) If for some positive integer $M$, the integers $M-1, M-2, M-4, M-$ $8, \ldots, M-2^{\left\lceil\log _{2} M\right\rceil-1}$ are all contained in the set $A$, then $\left\lceil\log _{2} M\right\rceil$ is odd and $M=2^{\left\lceil\log _{2} M\right\rceil}-1$.

Lemma 2.4. (See [6, Claim 4]) If for some positive integer $M$, the integers $M-1, M-2, M-4, M-$ $8, \ldots, M-2^{\left\lceil\log _{2} M\right\rceil-1}$ are all contained in the set $B$, then $\left\lceil\log _{2} M\right\rceil$ is even and $M=2^{\left\lceil\log _{2} M\right\rceil}-1$. 
Lemma 2.5. If for some even positive integer $M$, the integers $M-2, M-4, \ldots, M-2^{\left\lceil\log _{2} M\right\rceil-1}$ are all contained in the set $A$, then $M=2^{\left\lceil\log _{2} M\right\rceil}-2$.

Proof. If for some even positive integer $M$, the integers $M-2, M-4, \ldots, M-2^{\left\lceil\log _{2} M\right\rceil-1}$ are all contained in the set $A$, then $\frac{M}{2}-1, \frac{M}{2}-2, \ldots, \frac{M}{2}-2^{\left\lceil\log _{2} \frac{M}{2}\right\rceil-1}$ are all contained in the set $A$. By Lemma 2.3 , it follows that $\frac{M}{2}=2^{\left\lceil\log _{2} \frac{M}{2}\right\rceil}-1$, and so $M=2^{\left\lceil\log _{2} M\right\rceil}-2$.

Lemma 2.6. (See [6, Theorem 3]) Let $C$ and $D$ be sets of nonnegative integers such that $C \cup D=[0, m]$ and $C \cap D=\emptyset, 0 \in C$. Then $R_{C}(n)=R_{D}(n)$ for every positive integer $n$ if and only if there exists an nonnegative integer $l$ such that $C=A_{l}$ and $D=B_{l}$.

Lemma 2.7. (See [6, Corollary 1]) If $C=A \cap[0, m]$ and $D=B \cap[0, m]$, where $m$ is a positive integer not of the form $2^{l}-1$, then there exists a positive integer $m<n<2 m$ such that $R_{C}(n) \neq R_{D}(n)$.

\section{Proofs}

Proof of Theorem 1.1. (Sufficiency). Suppose that there exist a positive integer $l \geq 2$ such that $C=$ $A_{l-1} \cup\left(2^{l-1}+1+B_{l-1}\right)$ and $D=B_{l-1} \cup\left(2^{l-1}+1+A_{l-1}\right)$. Now we prove that $R_{C}(n)=R_{D}(n)$ for all nonnegative integers $n$. Clearly

$$
\begin{aligned}
R_{C}(n) & =\left|\left\{\left(c, c^{\prime}\right): c<c^{\prime}, c, c^{\prime} \in A_{l-1}, c+c^{\prime}=n\right\}\right|+\left|\left\{\left(c, c^{\prime}\right): c \in A_{l-1}, c^{\prime} \in 2^{l-1}+1+B_{l-1}, c+c^{\prime}=n\right\}\right| \\
& +\left|\left\{\left(c, c^{\prime}\right): c<c^{\prime}, c, c^{\prime} \in 2^{l-1}+1+B_{l-1}, c+c^{\prime}=n\right\}\right| \\
& =R_{A_{l-1}}(n)+\left|\left\{\left(c, c^{\prime}\right): c \in A_{l-1}, c^{\prime} \in B_{l-1}, c+c^{\prime}=n-\left(2^{l-1}+1\right)\right\}\right|+R_{B_{l-1}}\left(n-2\left(2^{l-1}+1\right)\right) .
\end{aligned}
$$

Similarly

$$
R_{D}(n)=R_{B_{l-1}}(n)+\left|\left\{\left(d, d^{\prime}\right): d \in B_{l-1}, d^{\prime} \in A_{l-1}, d+d^{\prime}=n-\left(2^{l-1}+1\right)\right\}\right|+R_{A_{l-1}}\left(n-2\left(2^{l-1}+1\right)\right) .
$$

By Lemma 2.1, for any positive integer $m, R_{A_{l-1}}(m)=R_{B_{l-1}}(m)$. Hence $R_{C}(n)=R_{D}(n)$.

(Necessity). Suppose that $C \cup D=[0, m] \backslash\{r\}, C \cap D=\emptyset$, and for any positive integer $n, R_{C}(n)=R_{D}(n)$. Let $C^{\prime}=m-C, D^{\prime}=m-D$. Then by Lemma 2.2, we have $C^{\prime} \cup D^{\prime}=[0, m] \backslash\{m-r\}, C^{\prime} \cap D^{\prime}=\emptyset$ and $R_{C^{\prime}}(n)=R_{D^{\prime}}(n)$ for any positive integer. Hence we assume that $r \leq \frac{m}{2}$ without loss of generality.

Let $p_{C}(x)=\sum_{i=0}^{m} \chi_{C}(i) x^{i}, p_{D}(x)=\sum_{i=0}^{m} \chi_{D}(i) x^{i}$. Since $C \cup \bar{D}=[0, m] \backslash\{r\}, C \cap D=\emptyset$, it follows that

$$
p_{C}(x)+p_{D}(x)=\frac{1-x^{m+1}}{1-x}-x^{r} .
$$

Hence $p_{D}(x)=\frac{1-x^{m+1}}{1-x}-p_{C}(x)-x^{r}$. Since $R_{C}(n)=R_{D}(n)$ for any positive integer $n$, we have

$$
\sum_{n=0}^{\infty} R_{C}(n) x^{n}=\sum_{n=0}^{\infty} R_{D}(n) x^{n}
$$

Hence

$$
\begin{aligned}
& \frac{1}{2} p_{C}(x)^{2}-\frac{1}{2} p_{C}\left(x^{2}\right)=\frac{1}{2}\left(\sum_{i \in C} x^{i}\right)^{2}-\frac{1}{2} \sum_{i \in C} x^{2 i}=\sum_{\substack{i_{1}, i_{2} \in C \\
i_{1}<i_{2}}} x^{i_{1}} \cdot x^{i_{2}} \\
= & \sum_{\substack{i_{1}, i_{2} \in C \\
i_{1}<i_{2}}} x^{i_{1}+i_{2}}=\sum_{n=0}^{\infty} R_{C}(n) x^{n}=\sum_{n=0}^{\infty} R_{D}(n) x^{n}=\frac{1}{2} p_{D}(x)^{2}-\frac{1}{2} p_{D}\left(x^{2}\right),
\end{aligned}
$$

and then

$$
p_{C}(x)^{2}-p_{C}\left(x^{2}\right)=\left(\frac{1-x^{m+1}}{1-x}-p_{C}(x)-x^{r}\right)^{2}-\left(\frac{1-x^{2 m+2}}{1-x^{2}}-p_{C}\left(x^{2}\right)-x^{2 r}\right)
$$




$$
\begin{aligned}
= & \left(\frac{1-x^{m+1}}{1-x}\right)^{2}+p_{C}(x)^{2}+x^{2 r}-2 \frac{1-x^{m+1}}{1-x} \cdot p_{C}(x)-2 \frac{1-x^{m+1}}{1-x} \cdot x^{r} \\
& +2 p_{C}(x) x^{r}-\frac{1-x^{2 m+2}}{1-x^{2}}+p_{C}\left(x^{2}\right)+x^{2 r} .
\end{aligned}
$$

Therefore,

$$
2 p_{C}\left(x^{2}\right)=\frac{1-x^{2 m+2}}{1-x^{2}}+2 p_{C}(x) \frac{1-x^{m+1}}{1-x}-\left(\frac{1-x^{m+1}}{1-x}\right)^{2}+2 x^{r} \frac{1-x^{m+1}}{1-x}-2 p_{C}(x) x^{r}-2 x^{2 r} .
$$

We first check the necessity for $1 \leq r \leq 5$.

For $r=1$, if there is a suitable decomposition $C \cup D=[0, m] \backslash\{1\}$ and $m \geq 4$, then $0 \in C$ and $2,3,4 \in D$. Let $E=m-C, F=m-D$. Then

$$
(E \cap[0, m-2]) \cap(F \cap[0, m-2])=\emptyset, \quad(E \cap[0, m-2]) \cup(F \cap[0, m-2])=[0, m-2] .
$$

By Lemma 2.2, $R_{E}(n)=R_{F}(n)$ for all integers $n \leq m-2$. By Lemma 2.1, $F \cap[0, m-2]=A \cap[0, m-2]$ or $B \cap[0, m-2]$, but this is a contradiction, because $F \cap[0, m-2]$ contains three consecutive integers $m-4$, $m-3$ and $m-2$. Hence the only solution is $m=2$.

For $r=2,3,4,5$, the proof is similar. Here we give the details in the case $r=4$. Since $0 \in C$, we have $1 \in D$, otherwise $R_{C}(1) \neq R_{D}(1)$. Similarly we can obtain $2 \in D, 3 \in C, 5 \in D, 6 \in C, 7 \in C, 8 \in D$, $9 \in D, 10 \in C, 11 \in D, 12 \in C, 13 \in D$, and we can not put 14 in both $C$ and $D$. Hence if $m \geq 14$, there is no $C$ and $D$. For $m<13$, we can check only $m=8$ holds and $C=\{0,3,6,7\}, D=\{1,2,5,8\}$.

Now we assume $r \geq 6$. First we shall prove that $r$ is even. Suppose that $r$ is odd. Let $k$ be an even integer such that $r<k<2 r$. Then the coefficient of $x^{k}$ in (3.1) is

$$
2 \chi_{C}\left(\frac{k}{2}\right)=1+2 \sum_{i \leq k} \chi_{C}(i)-(k+1)+2-2 \chi_{C}(k-r)
$$

and the coefficient of $x^{k-1}$ in (3.1) is

$$
0=0+2 \sum_{i \leq k-1} \chi_{C}(i)-k+2-2 \chi_{C}(k-1-r)
$$

Calculating $((3.2)-(3.3)) \times \frac{1}{2}$, we have

$$
\chi_{C}\left(\frac{k}{2}\right)=\chi_{C}(k)-\chi_{C}(k-r)+\chi_{C}(k-1-r)
$$

By Lemma 2.1, we have

$$
\begin{aligned}
& C \cap[0, r-1]=A \cap[0, r-1], \\
& D \cap[0, r-1]=B \cap[0, r-1] .
\end{aligned}
$$

Since $k-1-r$ is even, $k-r<r$, by (3.5) and the definition of $A$, we have

$$
\chi_{C}(k-r)+\chi_{C}(k-1-r)=1 .
$$

If $\chi_{C}(k-r)=0, \chi_{C}(k-1-r)=1$, then by $(3.4)$, we have $\chi_{C}(k)=0$ and $\chi_{C}\left(\frac{k}{2}\right)=1$.

If $\chi_{C}(k-r)=1, \chi_{C}(k-1-r)=0$, then by $(3.4)$, we have $\chi_{C}(k)=1$ and $\chi_{C}\left(\frac{k}{2}\right)=0$.

Hence $\chi_{C}(k-1-r)=\chi_{C}\left(\frac{k}{2}\right)$.

Let $k=2 r-2^{i+1}+2$, where $i \geq 1$. Then $\frac{k}{2}=r+1-2^{i}, k-1-r=r+1-2^{i+1}$. Hence $\chi_{C}\left(r+1-2^{i}\right)=$ $\chi_{C}\left(r+1-2^{i+1}\right)$. Therefore,

$$
\chi_{C}(r+1-2)=\chi_{C}(r+1-4)=\cdots=\chi_{C}\left(r+1-2^{\left\lceil\log _{2}(r+1)\right\rceil-1}\right),
$$


and so

$$
\chi_{A}(r+1-2)=\chi_{A}(r+1-4)=\cdots=\chi_{A}\left(r+1-2^{\left\lceil\log _{2}(r+1)\right\rceil-1}\right) .
$$

By Lemma 2.5, it follows that $r+1=2^{u}-2$ and $\chi_{A}(r+1)=\chi_{A}(r)$.

Next we shall prove $r \in B$. Suppose that $r \in A$. Since $0 \in C, r \notin C$, by (3.5), it follows that

$$
R_{C}(r)=R_{C \cap[0, r-1]}(r)=R_{A \cap[0, r-1]}(r)=R_{A \cap[0, r]}(r)-1 .
$$

Similarly, by (3.6), it follows that

$$
R_{D}(r)=R_{D \cap[0, r-1]}(r)=R_{B \cap[0, r-1]}(r)=R_{B \cap[0, r]}(r) .
$$

Noting that

$$
R_{A \cap[0, r]}(r)=R_{A}(r)=R_{B}(r)=R_{B \cap[0, r]}(r),
$$

we have $R_{C}(r) \neq R_{D}(r)$, a contradiction. Hence $r \in B$ and $r+1 \in B$, and so $r \notin A$.

By (3.5) and $r \notin C$, we have $C \cap[0, r]=A \cap[0, r]$. Hence

$$
R_{C}(r+1)=R_{C \cap[0, r]}(r+1)+\chi_{C}(r+1)=R_{A \cap[0, r]}(r+1)+\chi_{C}(r+1) .
$$

By (3.6) and $1, r \in B, r \notin D$, we have

$$
R_{D}(r+1)=R_{B \cap[0, r]}(r+1)-1 .
$$

Noting that $r, r+1 \in B$, we have

$$
R_{A \cap[0, r]}(r+1)=R_{A \cap[0, r+1]}(r+1), \quad R_{B \cap[0, r]}(r+1)=R_{B \cap[0, r+1]}(r+1) .
$$

Since

$$
R_{A \cap[0, r+1]}(r+1)=R_{A}(r+1)=R_{B}(r+1)=R_{B \cap[0, r+1]}(r+1),
$$

it follows that $R_{C}(r+1) \neq R_{D}(r+1)$, a contradiction.

Therefore, $r$ is not odd.

Suppose that $r$ is even. Take an even integer $k$ with $r \leq k \leq 2 r$. Then the coefficient of $x^{k-2}$ in (3.1) is

$$
2 \chi_{C}\left(\frac{k-2}{2}\right)=1+2 \sum_{i \leq k-2} \chi_{C}(i)-(k-1)+2-2 \chi_{C}(k-2-r),
$$

and the coefficient of $x^{k-1}$ in (3.1) is

$$
0=0+2 \sum_{i \leq k-1} \chi_{C}(i)-k+2-2 \chi_{C}(k-1-r) .
$$

Calculating $((3.9)-(3.10)) \times \frac{1}{2}$, we obtain

$$
\chi_{C}\left(\frac{k-2}{2}\right)=1-\chi_{C}(k-1)-\chi_{C}(k-2-r)+\chi_{C}(k-1-r) .
$$

By Lemma 2.2, $k-1-r<r$ and $k-2-r$ is even. By (3.5) and the definition of $A$,

$$
\chi_{C}(k-2-r)+\chi_{C}(k-1-r)=1 .
$$

If $\chi_{C}(k-2-r)=0$ and $\chi_{C}(k-1-r)=1$, then $\chi_{C}(k-1)=1, \chi_{C}\left(\frac{k-2}{2}\right)=1$.

If $\chi_{C}(k-2-r)=1, \chi_{C}(k-1-r)=0$, then $\chi_{C}(k-1)=0, \chi_{C}\left(\frac{k-2}{2}\right)=0$.

By the above two cases, we have $\chi_{C}(k-1-r)=\chi_{C}\left(\frac{k-2}{2}\right)$. Take

$$
k=2 r-2^{i+1}, i=0,1, \ldots,\left\lceil\log _{2}(r-1)\right\rceil-2,
$$


then $\frac{k-2}{2}=r-1-2^{i}, k-1-r=r-1-2^{i+1}$, and so $\chi_{C}\left(r-1-2^{i}\right)=\chi_{C}\left(r-1-2^{i+1}\right)$. Hence

$$
\chi_{C}(r-1-1)=\chi_{C}(r-1-2)=\cdots=\chi_{C}\left(r-1-2^{\left\lceil\log _{2}(r-1)\right\rceil-1}\right) .
$$

By lemma 2.4, we have $r-1=2^{\left\lceil\log _{2}(r-1)\right\rceil}-1$. Write $l=\left\lceil\log _{2}(r-1)\right\rceil+1$. Then $r=2^{l-1}$. Noting that $r \leq \frac{m}{2}$, then $[0,2 r] \subseteq[0, m]$. By Lemma 1 and the sufficiency of Theorem 1 , it follows that

$$
C \cap[0,2 r]=A_{l-1} \cup\left(2^{l-1}+1+B_{l-1}\right), \quad D \cap[0,2 r]=B_{l-1} \cup\left(2^{l-1}+1+A_{l-1}\right) .
$$

Next we shall prove $m=2 r$. First, we prove $m<3 \cdot 2^{l-1}=2^{l-1}+2^{l}$. Suppose that $m \geq 3 \cdot 2^{l-1}$. First we shall prove the following structure of $C$ and $D$ :

$$
\begin{aligned}
& C \cap\left[0,3 \cdot 2^{l-1}\right]=A_{l-1} \cup\left(2^{l-1}+1+B_{l-1}\right) \cup\left(2^{l}+1+B_{l-1}\right), \\
& D \cap\left[0,3 \cdot 2^{l-1}\right]=B_{l-1} \cup\left(2^{l-1}+1+A_{l-1}\right) \cup\left(2^{l}+1+A_{l-1}\right) .
\end{aligned}
$$

Write

$$
A_{l-1} \cup\left(2^{l-1}+1+B_{l-1}\right) \cup\left(2^{l}+1+B_{l-1}\right):=E, \quad B_{l-1} \cup\left(2^{l-1}+1+A_{l-1}\right) \cup\left(2^{l}+1+A_{l-1}\right):=F .
$$

If $n \leq 2^{l}$, then clearly we have $R_{E}(n)=R_{F}(n)$. Now we assume that $2^{l}<n \leq 3 \cdot 2^{l-1}$. It follows that

$$
\begin{aligned}
R_{E}(n) & =\left|\left\{\left(c, c^{\prime}\right): c \in A_{l-1}, c^{\prime} \in 2^{l-1}+1+B_{l-1}, c+c^{\prime}=n\right\}\right| \\
& +\left|\left\{\left(c, c^{\prime}\right): c \in A_{l-1}, c^{\prime} \in 2^{l}+1+B_{l-1}, c+c^{\prime}=n\right\}\right| \\
& +\left|\left\{\left(c, c^{\prime}\right): c<c^{\prime}, c, c^{\prime} \in 2^{l-1}+1+B_{l-1}, c+c^{\prime}=n\right\}\right| \\
& =\left|\left\{\left(c, c^{\prime}\right): c \in A_{l-1}, c^{\prime} \in B_{l-1}, c+c^{\prime}=n-\left(2^{l-1}+1\right)\right\}\right| \\
& +\mid\left\{\left(c, c^{\prime}\right): c \in A_{l-1}, c^{\prime} \in B_{l-1}, c+c^{\prime}=n-\left(2^{l}+1\right) \mid+R_{B_{l-1}}\left(n-2\left(2^{l-1}+1\right)\right)\right.
\end{aligned}
$$

and

$$
\begin{aligned}
R_{F}(n)= & \left|\left\{\left(d, d^{\prime}\right): d \in B_{l-1}, d^{\prime} \in 2^{l-1}+1+A_{l-1}, d+d^{\prime}=n\right\}\right| \\
& +\left|\left\{\left(d, d^{\prime}\right): d \in B_{l-1}, d^{\prime} \in 2^{l}+1+A_{l-1}, d+d^{\prime}=n\right\}\right| \\
& +\left|\left\{\left(d, d^{\prime}\right): d<d^{\prime}, d, d^{\prime} \in 2^{l-1}+1+B_{l-1}, d+d^{\prime}=n\right\}\right| \\
& =\left|\left\{\left(d, d^{\prime}\right): d \in B_{l-1}, d^{\prime} \in A_{l-1}, d+d^{\prime}=n-\left(2^{l-1}+1\right)\right\}\right| \\
& +\mid\left\{\left(d, d^{\prime}\right): d \in B_{l-1}, d^{\prime} \in A_{l-1}, d+d^{\prime}=n-\left(2^{l}+1\right) \mid+R_{A_{l-1}}\left(n-2\left(2^{l-1}+1\right)\right) .\right.
\end{aligned}
$$

By Lemma 2.6, $R_{A_{l-1}}(m)=R_{B_{l-1}}(m)$ holds for any positive integers $m$, and then $R_{E}(n)=R_{F}(n)$. By Lemma 2.1, $C \cap\left[0,3 \cdot 2^{l-1}\right]=E, D \cap\left[0,3 \cdot 2^{l-1}\right]=F$.

Next we shall prove that $m \geq 3 \cdot 2^{l-1}$ is impossible.

If $m>3 \cdot 2^{l-1}$, then by $0 \in C$ we have

$$
\begin{aligned}
R_{C}\left(3 \cdot 2^{l-1}+1\right) & =\chi_{C}\left(3 \cdot 2^{l-1}+1\right)+\left|\left\{\left(c, c^{\prime}\right): c \in A_{l-1}, c^{\prime} \in 2^{l}+1+B_{l-1}, c+c^{\prime}=3 \cdot 2^{l-1}+1\right\}\right| \\
& +\left|\left\{\left(c, c^{\prime}\right): c<c^{\prime}, c, c^{\prime} \in 2^{l-1}+1+B_{l-1}, c+c^{\prime}=3 \cdot 2^{l-1}+1\right\}\right| \\
& =\chi_{C}\left(3 \cdot 2^{l-1}+1\right)+\left|\left\{\left(c, c^{\prime}\right): c \in A_{l-1}, c^{\prime} \in B_{l-1}, c+c^{\prime}=2^{l-1}\right\}\right|+R_{B_{l-1}}\left(2^{l-1}-1\right)
\end{aligned}
$$

Similarly we have

$$
R_{D}\left(3 \cdot 2^{l-1}+1\right)=\left|\left\{\left(d, d^{\prime}\right): d \in B_{l-1}, d^{\prime} \in A_{l-1}, d+d^{\prime}=2^{l-1}\right\}\right|+R_{A_{l-1}}\left(2^{l-1}-1\right) .
$$

By Lemma 2.6, $R_{A_{l-1}}\left(2^{l-1}-1\right)=R_{B_{l-1}}\left(2^{l-1}-1\right)$. Hence $R_{C}\left(3 \cdot 2^{l-1}+1\right)=R_{D}\left(3 \cdot 2^{l-1}+1\right)$ if and only if $\chi_{C}\left(3 \cdot 2^{l-1}+1\right)=0$, that is, $3 \cdot 2^{l-1}+1 \in D$.

Since $1,3 \cdot 2^{l-1}+1,2^{l}+1,2^{l-1}+1 \in D$ and

$$
3 \cdot 2^{l-1}+2=1+\left(1+3 \cdot 2^{l-1}\right)=\left(2^{l-1}+1\right)+\left(2^{l}+1\right),
$$


it follows that

$$
\begin{aligned}
& R_{D}\left(3 \cdot 2^{l-1}+2\right)=1+1+\left|\left\{\left(d, d^{\prime}\right): d \in B_{l-1}, d^{\prime} \in 2^{l}+1+A_{l-1}, d+d^{\prime}=3 \cdot 2^{l-1}+2\right\}\right| \\
& +\left|\left\{\left(d, d^{\prime}\right): d<d^{\prime}, d, d^{\prime} \in 2^{l-1}+1+A_{l-1}, d+d^{\prime}=3 \cdot 2^{l-1}+2\right\}\right| \\
& =1+1+\mid\left\{\left(d, d^{\prime}\right): d \in B_{l-1}, d^{\prime} \in A_{l-1}, d+d^{\prime}=2^{l-1}+1 \mid+R_{A_{l-1}}\left(2^{l-1}\right),\right.
\end{aligned}
$$

Similarly,

$$
R_{C}\left(3 \cdot 2^{l-1}+2\right)=\chi_{C}\left(3 \cdot 2^{l-1}+2\right)+\left|\left\{\left(c, c^{\prime}\right): c \in A_{l-1}, c^{\prime} \in B_{l-1}, c+c^{\prime}=2^{l-1}+1\right\}\right|+R_{B_{l-1}}\left(2^{l-1}\right) .
$$

Hence $R_{C}\left(3 \cdot 2^{l-1}+2\right) \neq R_{D}\left(3 \cdot 2^{l-1}+2\right)$, a contradiction.

If $m=3 \cdot 2^{l-1}$, by $3 \cdot 2^{l-1}+2=\left(2^{l-1}+1\right)+\left(2^{l}+1\right)$, then we have

$$
\begin{aligned}
& R_{C}\left(3 \cdot 2^{l-1}+2\right)=\mid\left\{\left(c, c^{\prime}\right): c \in A_{l-1}, c^{\prime} \in B_{l-1}, c<c^{\prime}, c+c^{\prime}=2^{l-1}+1 \mid+R_{B_{l-1}}\left(2^{l-1}\right),\right. \\
& R_{D}\left(3 \cdot 2^{l-1}+2\right)=1+\mid\left\{\left(d, d^{\prime}\right): d \in B_{l-1}, d^{\prime} \in A_{l-1}, d<d^{\prime}, d+d^{\prime}=2^{l-1}+1 \mid+R_{A_{l-1}}\left(2^{l-1}\right) .\right.
\end{aligned}
$$

Hence $R_{C}\left(3 \cdot 2^{l-1}+2\right) \neq R_{D}\left(3 \cdot 2^{l-1}+2\right)$, a contradiction.

Therefore, $m<3 \cdot 2^{l-1}$.

Now we have

$$
\begin{aligned}
& C=A_{l-1} \cup\left(2^{l-1}+1+B_{l-1}\right) \cup\left(2^{l}+1+\left(B_{l-1} \cap\left[0, m-\left(2^{l}+1\right)\right]\right)\right), \\
& D=B_{l-1} \cup\left(2^{l-1}+1+A_{l-1}\right) \cup\left(2^{l}+1+\left(A_{l-1} \cap\left[0, m-\left(2^{l}+1\right)\right]\right)\right) .
\end{aligned}
$$

Next we prove $m=2^{l}$. Suppose that $m>2^{l}$.

If $m-\left(2^{l}+1\right) \neq 2^{k}-1$, where $k$ is a nonnegative integer. By Lemma 2.7, there exists an integer $u$ satisfying $m-\left(2^{l}+1\right)<u<2\left[m-\left(2^{l}+1\right)\right]$ such that

$$
R_{A \cap\left[0, m-\left(2^{l}+1\right)\right]}(u)=R_{A_{l-1} \cap\left[0, m-\left(2^{l}+1\right)\right]}(u) \neq R_{B_{l-1} \cap\left[0, m-\left(2^{l}+1\right)\right]}(u)=R_{B \cap\left[0, m-\left(2^{l}+1\right)\right]}(u) .
$$

Since $m+\left(2^{l}+1\right)<u+2\left(2^{l}+1\right)<2 m$, it follows that

$$
\begin{aligned}
& R_{C}\left(2\left(2^{l}+1\right)+u\right)=R_{2^{l}+1+\left(B_{l-1} \cap\left[0, m-\left(2^{l}+1\right)\right]\right)}\left(2\left(2^{l}+1\right)+u\right) \\
= & R_{\left.B_{l-1} \cap\left[0, m-\left(2^{l}+1\right)\right]\right)}(u)=R_{B \cap\left[0, m-\left(2^{l}+1\right)\right]}(u) .
\end{aligned}
$$

Similarly

$$
\begin{aligned}
& R_{D}\left(2\left(2^{l}+1\right)+u\right)=R_{2^{l}+1+\left(A_{l-1} \cap\left[0, m-\left(2^{l}+1\right)\right]\right)}\left(2\left(2^{l}+1\right)+u\right) \\
= & R_{\left.A_{l-1} \cap\left[0, m-\left(2^{l}+1\right)\right]\right)}(u)=R_{A \cap\left[0, m-\left(2^{l}+1\right)\right]}(u) .
\end{aligned}
$$

Since $R_{C}\left(2\left(2^{l}+1\right)+u\right)=R_{D}\left(2\left(2^{l}+1\right)+u\right)$, it follows that $R_{A \cap\left[0, m-\left(2^{l}+1\right)\right]}(u)=R_{B \cap\left[0, m-\left(2^{l}+1\right)\right]}(u)$, a contradiction with (3.11).

Now we suppose that $m-\left(2^{l}+1\right)=2^{k}-1$. Noting that $m<3 \cdot 2^{l-1}$, we have $k<l-1$. Hence

$$
\begin{aligned}
& C=A_{l-1} \cup\left(2^{l-1}+1+B_{l-1}\right) \cup\left(2^{l}+1+B_{k}\right) \\
& D=B_{l-1} \cup\left(2^{l-1}+1+A_{l-1}\right) \cup\left(2^{l}+1+A_{k}\right) .
\end{aligned}
$$

If $k=0$, then $2^{l}+1 \in D$. Now we divide the following two cases.

Case 1. $2^{l-1}-1 \in A_{l-1}$. That is, $2^{l} \in D$. Hence $2^{l+1}+1=\left(2^{l}+1\right)+2^{l}$, and so $R_{C}\left(2^{l+1}+1\right)=0$, $R_{D}\left(2^{l+1}+1\right)=1$, a contradiction.

Case 2. $2^{l-1}-1 \in B_{l-1}$. That is, $2^{l} \in C$. Hence $2^{l-1}-2 \in A_{l-1}$, and so $2^{l}-1 \in D$. Since $2^{l+1}=\left(2^{l}+1\right)+\left(2^{l}-1\right)$, it follows that $R_{C}\left(2^{l+1}\right)=0, R_{D}\left(2^{l+1}\right)=1$, a contradiction.

Therefore, we assume that $k>0$. Let $C^{\prime}=2^{l}+2^{k}-C, D^{\prime}=2^{l}+2^{k}-D$.

Case 1. $2 \nmid l-1,2 \nmid k$. It follows that

$$
\begin{aligned}
& C^{\prime}=A_{k} \cup\left(2^{k}+A_{l-1}\right) \cup\left(2^{l-1}+2^{k}+1+B_{l-1}\right), \\
& D^{\prime}=B_{k} \cup\left(2^{k}+B_{l-1}\right) \cup\left(2^{l-1}+2^{k}+1+A_{l-1}\right) .
\end{aligned}
$$


Case 2. $2 \nmid l-1,2 \mid k$. Then

$$
\begin{aligned}
& C^{\prime}=B_{k} \cup\left(2^{k}+A_{l-1}\right) \cup\left(2^{l-1}+2^{k}+1+B_{l-1}\right), \\
& D^{\prime}=A_{k} \cup\left(2^{k}+B_{l-1}\right) \cup\left(2^{l-1}+2^{k}+1+A_{l-1}\right) .
\end{aligned}
$$

Case 3. $2 \mid l-1,2 \nmid k$. Then

$$
\begin{aligned}
& C^{\prime}=A_{k} \cup\left(2^{k}+B_{l-1}\right) \cup\left(2^{l-1}+2^{k}+1+A_{l-1}\right) \\
& D^{\prime}=B_{k} \cup\left(2^{k}+A_{l-1}\right) \cup\left(2^{l-1}+2^{k}+1+B_{l-1}\right) .
\end{aligned}
$$

Case 4. $2|l-1,2| k$. Then

$$
\begin{aligned}
& C^{\prime}=B_{k} \cup\left(2^{k}+B_{l-1}\right) \cup\left(2^{l-1}+2^{k}+1+A_{l-1}\right), \\
& D^{\prime}=A_{k} \cup\left(2^{k}+A_{l-1}\right) \cup\left(2^{l-1}+2^{k}+1+B_{l-1}\right) .
\end{aligned}
$$

Hence $C^{\prime} \cup D^{\prime}=\left[0,2^{l}+2^{k}\right] \backslash\left\{2^{l-1}+2^{k}\right\}, C^{\prime} \cap D^{\prime}=\emptyset$. By Lemma 2.2, it follows that $R_{C^{\prime}}(n)=R_{D^{\prime}}(n)$ for all positive integers $n$. By Lemma 2.1, it suffices to consider the following two cases.

(I) $C^{\prime} \cap\left[0,2^{l-1}+2^{k}-1\right]=A \cap\left[0,2^{l-1}+2^{k}-1\right]$ and $D^{\prime} \cap\left[0,2^{l-1}+2^{k}-1\right]=B \cap\left[0,2^{l-1}+2^{k}-1\right]$.

(II) $C^{\prime} \cap\left[0,2^{l-1}+2^{k}-1\right]=B \cap\left[0,2^{l-1}+2^{k}-1\right]$ and $D^{\prime} \cap\left[0,2^{l-1}+2^{k}-1\right]=A \cap\left[0,2^{l-1}+2^{k}-1\right]$.

If Case 1 and Case (I) hold, then $C^{\prime} \cap\left[0,2^{k}-1\right]=A \cap\left[0,2^{k}-1\right]=A_{k}$. By the property of $A$, we have $C^{\prime} \cap\left[2^{k}, 2^{k+1}-1\right]=2^{k}+B_{k}$. By Case 1 , we have $C^{\prime} \cap\left[2^{k}, 2^{k+1}-1\right]=2^{k}+A_{k}$, a contradiction.

If Case 1 and Case (II) hold, then $C^{\prime} \cap\left[0,2^{k}-1\right]=B \cap\left[0,2^{k}-1\right]=B_{k}$. By Case 1 , we have $C^{\prime} \cap$ $\left[0,2^{k}-1\right]=A_{k}$, a contradiction.

If Case 2 and Case (I) hold, then $C^{\prime} \cap\left[0,2^{k}-1\right]=A_{k}$. By Case 2, we have $C^{\prime} \cap\left[0,2^{k}-1\right]=B_{k}$, a contradiction.

If Case 2 and Case (II) hold, then $k<l-1$, and so $2^{k}+1<2^{l-1}-1$. By the property of $A$, it follows that $2^{k}+1 \in A_{l-1}$, and then $2^{k}+\left(2^{k}+1\right) \in 2^{k}+A_{l-1} \subseteq C^{\prime}$. Hence $2^{k}+\left(2^{k}+1\right) \in C^{\prime}$. By Case (II), $2^{k}+\left(2^{k}+1\right) \in B$. But $2^{k}+\left(2^{k}+1\right)=2^{k+1}+1 \in A$, a contradiction.

If Case 3 and Case (I) hold, then by $2^{k}+1 \in A_{l-1}$, we have $2^{k}+\left(2^{k}+1\right) \in 2^{k}+A_{l-1} \subseteq D^{\prime}$. By Case (I), $2^{k}+\left(2^{k}+1\right) \in B$. However, by the property of $A, 2^{k}+\left(2^{k}+1\right)=2^{k+1}+1 \in A$, a contradiction.

If Case 3 and Case (II) hold, then $C^{\prime} \cap\left[0,2^{k}-1\right]=B \cap\left[0,2^{k}-1\right]=B_{k}$. By Case $3, C^{\prime} \cap\left[0,2^{k}-1\right]=A_{k}$, a contradiction.

If Case 4 and Case (I) hold, then $C^{\prime} \cap\left[0,2^{k}-1\right]=A_{k}$. By Case $4, C^{\prime} \cap\left[0,2^{k}-1\right]=B_{k}$, a contradiction.

If Case 4 and Case (II) hold, then $C^{\prime} \cap\left[0,2^{k}-1\right]=B \cap\left[0,2^{k}-1\right]=B_{k}$. By the property of $B$, $C^{\prime} \cap\left[2^{k}, 2^{k+1}-1\right]=2^{k}+A_{k}$. By Case $4, C^{\prime} \cap\left[2^{k}, 2^{k+1}-1\right]=2^{k}+B_{k}$, a contradiction.

Therefore, $m=2 r$.

\section{References}

[1] Y.-G. Chen, V.F. Lev, Integer sets with identical representation functions, Integers 16 (2016) A36.

[2] S.-Q. Chen, Y.-G. Chen, Integer sets with identical representation functions, II 94 (2021) No. 103293.

[3] S.-Q. Chen, M. Tang, Q.-H. Yang, On a problem of Chen and Lev, Bull. Aust. Math. Soc. 99 (2019) $15-22$.

[4] Y.-G. Chen, B. Wang, On additive properties of two special sequences, Acta Arith. 110 (2003) 299-303.

[5] G. Dombi, Additive properties of certain sets, Acta Arith. 103 (2002) 137-146.

[6] S.Z. Kiss, C. Sándor, Partitions of the set of nonnegative integers with the same representation functions, Discrete Math. 340 (2017) 1154-1161. 
[7] V.F. Lev, Reconstructing integer sets from their representation functions, Electron. J. Combin. 11 (2004) R78.

[8] J.-W. Li, M. Tang, Partitions of the set of nonnegative integers with the same representation functions, Bull. Aust. Math. Soc. 97 (2018) 200-206.

[9] C. Sándor, Partitions of natural numbers and their representation functions, Integers 4 (2004) A18.

[10] M. Tang, Partitions of the set of natural numbers and their representation functions, Discrete Math. 308 (2008) 2614-2616.

[11] M. Tang, Partitions of natural numbers and their representation functions, Chinese Ann. Math. Ser. A 37 (2016) 41-46; For English version, see, Chinese J. Contemp. Math. 37 (2016) 39-44.

[12] Q.-H. Yang, Y.-G. Chen, Partitions of natural numbers with the same weighted representation functions, J. Number Theory 132 (2012) 3047-3055.

[13] Q.-H. Yang, M. Tang, Representation functions on finite sets with extreme symmetric differences, J. Number Theory 180 (2017) 73-85.

[14] W. Yu, M. Tang, A note on partitions of natural numbers and their representation functions, Integers 12 (2012) A53. 